\title{
Momentum and Heat Transfer in a Laminar Boundary Layer with Slip Flow
}

\author{
Michael J. Martin* and Iain D. Boyd ${ }^{\dagger}$ \\ University of Michigan, Ann Arbor, Michigan 48109-2104
}

DOI: $10.2514 / 1.22968$

\begin{abstract}
Flow in a laminar boundary layer is modeled using a slip boundary condition. The slip condition changes the boundary layer structure from a self-similar profile to a two-dimensional structure. Although the slip condition generally leads to decreased overall drag, two-dimensional effects cause local increases in skin friction. Other effects include thinner boundary layers, delayed transition to turbulence, and changes in the heat transfer at the wall. Without a thermal jump condition, slip will lead to increased heat transfer. When a thermal jump boundary condition is added to simulate real gases, the heat transfer decreases to below the no-slip values.
\end{abstract}

\section{Nomenclature}

$\begin{array}{lll}a & = & \text { iteration coefficient } \\ b & = & \text { iteration coefficient } \\ C_{D} & = & \text { drag coefficient } \\ c_{p} & = & \text { specific heat ratio } \\ F_{D} & = & \text { drag force } \\ f & = & \text { nondimensional stream function } \\ i & = & \text { index in } K_{1} \text { direction } \\ j & = & \text { index in } \eta \text { direction } \\ K_{1} & = & \text { nonequilibrium parameter } \\ K n & = & \text { Knudsen number } \\ L & = & \text { length } \\ N u & = & \text { Nusselt number } \\ n & = & \text { distance in tangential direction } \\ P & = & \text { static pressure } \\ q^{\prime \prime} & = & \text { heat flux } \\ R e & = & \text { Reynolds number } \\ s & = & \text { distance in normal direction } \\ T & = & \text { temperature } \\ u & = & \text { velocity in the } x \text { direction } \\ v & = & \text { velocity in the } y \text { direction } \\ x & = & \text { position in flow normal direction } \\ y & = & \text { position in flow tangential direction } \\ \alpha & = & \text { thermal conductivity } \\ \beta & = & \text { slip length } \\ \gamma & = & \text { specific heat ratio } \\ \Delta & = & \text { step size } \\ \delta & = & \text { boundary layer thickness } \\ \delta^{*} & = & \text { displacement thickness } \\ \eta & = & \text { nondimensional position } \\ \eta & = & \text { nondimensional boundary layer thickness } \\ \theta & = & \text { momentum thickness } \\ \lambda & = & \text { mean free path } \\ \mu & = & \text { viscosity } \\ \rho & = & \text { density } \\ \sigma & = & \text { accommodation coefficient } \\ \tau & = & \text { shear stress } \\ v & = & \text { inematic viscosity } \\ & & \\ & & \end{array}$

Received 4 February 2006; revision received 12 May 2006; accepted for publication 14 May 2006. Copyright (c) 2006 by the American Institute of Aeronautics and Astronautics, Inc. All rights reserved. Copies of this paper may be made for personal or internal use, on condition that the copier pay the $\$ 10.00$ per-copy fee to the Copyright Clearance Center, Inc., 222 Rosewood Drive, Danvers, MA 01923; include the code $\$ 10.00$ in correspondence with the CCC.

${ }^{*}$ Graduate Student Research Assistant, Department of Aerospace Engineering. AIAA Student Member.

Professor, Department of Aerospace Engineering. AIAA Associate Fellow.

$$
\begin{array}{lll}
\text { Subscripts } & \\
g & = & \text { gas } \\
L & = & \text { length } \\
M & = & \text { momentum } \\
o & = & \text { freestream } \\
\text { slip } & = & \text { property of gas or liquid at wall } \\
T & = & \text { thermal } \\
x & = & \text { position on length of plate } \\
\delta & = & \text { boundary layer thickness }
\end{array}
$$

Superscripts

* $\quad=$ nondimensional value

, $=$ derivative with respect to $\eta$

" $=$ second derivative with respect to $\eta$

\section{Introduction}

A $S$ the number of applications of microelectromechanical systems (MEMS) increases, the study of gas and liquid flows at the microscale has become a topic of increasing interest [1,2]. In both gas and liquid phases, these flows are often dominated by surface interactions, making correct modeling of the wall boundary conditions essential. Slightly rarefied gas flows, with Knudsen numbers less than 0.1 , are modeled using the Maxwell slip condition [3]:

$$
u_{\text {slip }}=u_{g}-u_{w}=\left.\lambda \frac{2-\sigma_{M}}{\sigma_{M}} \frac{\partial u}{\partial n}\right|_{\text {wall }}+\left.\frac{3}{4} \frac{\mu}{\rho T_{g}} \frac{\partial T}{\partial s}\right|_{\text {wall }}
$$

where $u_{\text {slip }}$ is the wall slip velocity, $u_{g}$ is the gas velocity at the wall, $u_{w}$ is the wall velocity, $\partial u / \partial n$ is velocity gradient normal to the wall, $\sigma_{M}$ is the tangential momentum accommodation coefficient, $T_{g}$ is the temperature of the gas, and $\partial T / \partial s$ is the temperature gradient along the wall.

For an isothermal wall, this simplifies to

$$
u_{\text {slip }}^{*}=\left.K n \frac{2-\sigma_{M}}{\sigma_{M}} \frac{\partial u^{*}}{\partial n^{*}}\right|_{\text {wall }}
$$

where $K n$ is the Knudsen number at the wall, $u^{*}$ is the nondimensional velocity, and $n^{*}$ is the nondimensional distance in the wall normal direction.

Recent research [4] suggests that as length scales in liquid flows approach the continuum limit, a similar slip condition may apply. This slip condition is given as

$$
u_{\text {slip }}=\left.\beta \frac{\partial u}{\partial n}\right|_{\text {wall }}
$$


where $\beta$ is the slip length for the liquid. Although experimental measurements [5] suggest that the slip length for hydrophobic surfaces with water are on the order of $1 \mu \mathrm{m}$, the existence of liquid slip, as well as the appropriate length scales for $\beta$, is still the subject of controversy within the fluid mechanics community [6].

A first-order solution, based on integral methods, of the boundary layer equations suggests that the boundary layer thins as a result of slip effects [7]. However, integral methods were unable to show any changes in skin friction. An expanded version of this analysis suggested that heat transfer was unaffected by the appearance of velocity slip [8]. Computational studies have also been made of external flow conditions, such as gas flows over flat plate airfoils [9]. These studies have shown that nonequilibrium effects will change the skin friction on a flat plate in slip flow.

This paper provides a semi-analytic solution to the boundary layer equations for a flat plate under slightly rarefied flow conditions. The changes to the flow structure, including velocity profile and boundary layer thickness, are analyzed. The changes in viscous drag and heat transfer for the flat plate are calculated and correlated as functions of flow properties.

\section{Fluid Flow in a Laminar Boundary Layer with Slip}

\section{A. Formulation of Boundary Layer Equations with Slip Flow}

As shown in Fig. 1, flow over a plate can be described as consisting of a uniform external flow region and a boundary layer of finite thickness.

The flow is governed by the continuity equation and conservation of momentum in the $x$ direction:

$$
\begin{gathered}
\frac{\partial \rho}{\partial t}+\frac{\partial \rho u}{\partial x}+\frac{\partial \rho v}{\partial y}=0 \\
\frac{\partial u}{\partial t}+u \frac{\partial u}{\partial x}+v \frac{\partial u}{\partial y}=-\frac{\partial P}{\partial x}+v\left[\frac{\partial^{2} u}{\partial x^{2}}+\frac{\partial^{2} u}{\partial y^{2}}\right]
\end{gathered}
$$

This flow was first studied extensively by Blasius [10] who assumed steady, incompressible, laminar flow, no significant gradients of pressure in the $x$ direction, and that velocity gradients in the $x$ direction are small compared to velocity gradients in the $y$ direction. Based on these assumptions, Eqs, (4) and (5) then simplify into the boundary layer equations given as

$$
\begin{gathered}
\frac{\partial u}{\partial x}+\frac{\partial v}{\partial y}=0 \\
u \frac{\partial u}{\partial x}+v \frac{\partial u}{\partial y}=v \frac{\partial^{2} u}{\partial y^{2}}
\end{gathered}
$$

These equations can then be transformed, using the nondimensionalizations and nondimensional stream functions given as

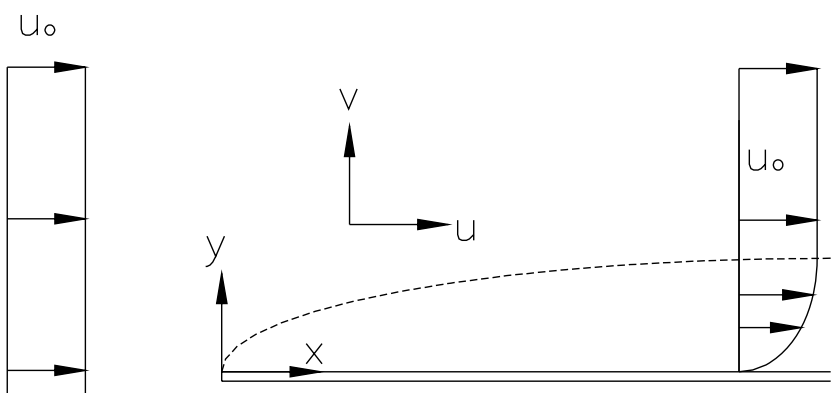

Fig. 1 Boundary layer flow over a flat plate.

$$
\begin{gathered}
x^{*}=x / L \\
y^{*}=\frac{y}{\left(v L / u_{o}\right)^{1 / 2}} \\
u^{*}=u / u_{o} \\
v^{*}=\frac{v}{\left(v u_{o} / x\right)^{1 / 2}} \\
\frac{y^{*}}{\left(x^{*}\right)^{1 / 2}}=\frac{y /\left(v L / u_{o}\right)^{1 / 2}}{(x / L)^{1 / 2}}=\frac{y}{\left(v x / u_{o}\right)^{1 / 2}} \\
u^{*}=\frac{u}{u_{o}}=f^{\prime}(\eta) \\
v \\
v^{*}=\frac{\left.v_{0} / x\right)^{1 / 2}}{\left(v u_{0}\right.}=0.5\left[\eta f^{\prime}(\eta)-f(\eta)\right]
\end{gathered}
$$

where $L$ is the length of the flat plate.

A governing equation for $f$ can be found by substituting these results into the $x$ momentum Eq. (7):

$$
f^{\prime \prime \prime}(\eta)=-0.5 f(\eta) f^{\prime \prime}(\eta)
$$

For flow at nonrarefied length scales, the boundary conditions for the problem are no slip, and no through flow at the wall, and $u$ is equal to the freestream velocity as $y$ approaches infinity. In nondimensional variables, these become

$$
\begin{gathered}
u^{*}(y=0)=0 \Rightarrow f^{\prime}(\eta=0)=0 \\
v^{*}(y=0)=0 \Rightarrow f(\eta=0)=0 \\
u^{*}(y \rightarrow \infty)=1 \Rightarrow f^{\prime}(\eta \rightarrow \infty)=1
\end{gathered}
$$

Based on these boundary conditions, Blasius was able to solve the problem using a shooting method, which gave an initial value of

$$
f^{\prime \prime}(\eta=0)=0.33206
$$

Using this result, Blasius calculated the self-similar laminar boundary layer, with a velocity profile and nondimensional shear stress.

When gas flow approaches the continuum limit, the no-slip condition given by Eq. (16) is replaced by the slip-flow condition given as Eq. (2). If we use the nondimensionalizations given in Eqs. (8-14), this condition can be nondimensionalized to obtain

$$
f^{\prime}(0)=\frac{\left(2-\sigma_{M}\right)}{\sigma_{M}} K n_{x} R e_{x}^{1 / 2} f^{\prime \prime}(0)=K_{1} f^{\prime \prime}(0)
$$

where $K n_{x}$ and $R e_{x}$ are the Knudsen and Reynolds numbers based on $x$, and $K_{1}$ is a nondimensional parameter that describes the behavior at the surface:

$$
K_{1}=\frac{\left(2-\sigma_{M}\right)}{\sigma_{M}} K n_{x} R e_{x}^{1 / 2}
$$

When liquid flows approach the continuum limit, an alternate definition of $K_{1}$ based on the slip length can be derived using the same nondimensionalizations:

$$
K_{1}=\frac{\beta}{x} R e_{x}^{1 / 2}
$$

The revised boundary condition suggests that self-similarity will be lost, and the velocity will be a function of both $\eta$ and $K_{1}$. Although the definition of $u^{*}$ is unchanged, the definition of $v^{*}$ must be 
modified to incorporate the derivative of the stream function with respect to $K_{1}$ :

$$
v^{*}=\frac{v}{(v u o / x)^{1 / 2}}=\frac{1}{2}\left[\eta \frac{\partial f\left(\eta, K_{1}\right)}{\partial \eta}-f\left(\eta, K_{1}\right)+K_{1} \frac{\partial f\left(\eta, K_{1}\right)}{\partial K_{1}}\right]
$$

When all other derivatives in $x$ are rewritten to include a $K_{1}$ term, the ordinary differential equation given in Eq. (15) is replaced by a partial differential equation:

$$
\frac{\partial^{3} f}{\partial \eta^{3}}=-0.5 f \frac{\partial^{2} f}{\partial \eta^{2}}-0.5 K_{1} \frac{\partial}{\partial \eta}\left[\frac{\partial f}{\partial \eta} \frac{\partial f}{\partial K_{1}}\right]
$$

The no-slip governing equation will be recovered when $K_{1}$ is equal to zero. At large values of $K_{1}$, Eq. (24) faces three distinct problems: the emergence of a singularity, the breakdown of the boundary layer assumptions, and the breakdown of the NavierStokes equations. Each of these phenomena should be considered in greater depth.

Large values of $K_{1}$ correspond to small values of $x$, or the leading edge of the plate. Because the definition of $\eta$ given in Eq. (12) is inversely proportion to the square root of $x$, the boundary layer equations approach a singularity as $x$ approaches zero, corresponding to $K_{1}$ approaching infinity.

Even before nondimensionaliziation, Eq. (7) is not accurate at the leading edge of the plate. The boundary layer equations are formulated on the assumption that the velocity gradients in the $x$ direction are small compared to velocity gradients in the $y$ direction. At the leading edge of the plate, this assumption is no longer true, leading to formation of a Stokes flow region [11]. Correct use of the boundary layer equations requires that this region be relatively small, which requires a Reynolds number of 500 or greater.

For gas flows at values of $K_{1}$ that correspond to a Knudsen number of 0.1 or greater, the Navier-Stokes equations break down, and Eq. (24) is no longer an accurate physical description of the flow. However, this region is smaller than the Stokes flow region described above and can be disregarded so long as larger values of Reynolds numbers, and smaller values of $K_{1}$, are achieved downstream.

For all of these reasons, this formulation of the boundary layer equations is susceptible to error at large values of $K_{1}$. However, just as when the no-slip boundary layer is integrated, the error in the leading edge can often be disregarded. When the values are then integrated over $x$ to provide quantities such as drag and heat transfer on the body, the contribution of the free-molecular and transition regions, and the error that is introduced in these areas, will be relatively small compared to the total integral.

To solve in the slip domain, the partial differential equation must be solved over large regions of $K_{1}$, including the entire slip-flow region, as well as into the transitional and free-molecular flow regions. This can be accomplished using a marching code, beginning from large values of $K_{1}$, and marching the code until $K_{1}$ approaches zero. Because large values of $K_{1}$ correspond to small values of $x$, this approach marches in the flow direction, similar to existing boundary layer codes [11].

\section{B. Numerical Solution of Fluid Flow in Boundary Layer Equations with Slip Flow}

Equation (24) is discretized using center-difference approximations for all derivatives with respect to $\eta$. To simplify the expression, $f^{\prime}$ is substituted for $\partial f / \partial \eta$ :

$$
f_{i, j}^{\prime}=\frac{f_{i, j+1}-f_{i, j-1}}{2 \Delta \eta}+O\left[(\Delta \eta)^{2}\right]
$$

Equation (24) then becomes

$$
\frac{\partial^{2} f^{\prime}}{\partial \eta^{2}}=-0.5 f \frac{\partial f^{\prime}}{\partial \eta}-0.5 K_{1} \frac{\partial}{\partial \eta}\left[f^{\prime} \frac{\partial f}{\partial K_{1}}\right]
$$

For the mixed derivative, a first-order upwind expression is used, as shown in Eq. (27):

$$
\begin{aligned}
& \frac{\partial}{\partial \eta}\left[f^{\prime} \frac{\partial f}{\partial K_{1}}\right]_{i, j}=\frac{1}{\Delta \eta} \frac{1}{\Delta K_{1}}\left[f_{i, j}^{\prime}\left(f_{i, j}-f_{i-1, j}\right)-f_{i, j-1}^{\prime}\left(f_{i, j-1}\right.\right. \\
& \left.\left.\quad-f_{i-1, j-1}\right)\right]
\end{aligned}
$$

This yields the following expression for $f_{i-1, j}$ :

$$
\begin{aligned}
& f_{i-1, j}=f_{i, j}+\frac{f_{i, j-1}^{\prime}}{f_{i, j}^{\prime}}\left(f_{i-1, j-1}-f_{i, j-1}\right) \\
& +\frac{2 \Delta K_{1}}{K_{1} f_{i, j}^{\prime}}\left(\frac{f_{i, j+1}^{\prime}-2.0 f_{i, j}^{\prime}+f_{i, j-1}^{\prime}}{\Delta \eta}+\frac{f_{i, j}}{4.0}\left(f_{i, j+1}^{\prime}-f_{i, j-1}^{\prime}\right)\right)
\end{aligned}
$$

When $K_{1}$ is large, flow is uniform in the $x$ direction, giving the initial conditions:

$$
\begin{aligned}
& f^{\prime}\left(K_{1} \rightarrow \infty\right)=1 \\
& f\left(K_{1} \rightarrow \infty\right)=\eta
\end{aligned}
$$

Along the wall, the boundary conditions are the no-through-flow condition given by Eq. (17) and the slip condition given by Eq. (20). The slip condition is implemented through the following first-order approximation:

$$
f_{i, 1}^{\prime}=\frac{K_{1 i} f_{i, 2}^{\prime}}{\left(K_{1 i}+\Delta \eta_{\text {wall }}\right)}+O\left(\frac{(\Delta \eta)^{2}}{\left(\Delta \eta+K_{1 i}\right)}\right) \frac{\partial^{2} f^{\prime}}{\partial \eta^{2}}
$$

The proposed scheme is conditionally stable. The following stability criteria apply:

$$
\begin{gathered}
\Delta K_{1} \leq \frac{7(\Delta \eta)^{2} K_{1}}{3} \\
\Delta K_{1} \leq \frac{\left(1+f_{i, j}^{\prime}\right) \Delta \eta K_{1}}{2 f_{i, j}}
\end{gathered}
$$

From the initial conditions at large $K_{1}$ specified in Eqs. (29) and (30), a maximum step size $\Delta K_{1}$ is calculated using Eqs. (32) and (33). The stream function at the next step is calculated explicitly using Eq. (28), and values of $f^{\prime}$ calculated using Eq. (25). Because the maximum step size $\Delta K_{1}$ becomes prohibitively small as $K_{1}$ approaches zero, the calculation is halted when it reaches a value of $K_{1}$ of less than 0.005 . The solution at $K_{1}$ equal to zero is then calculated using the classical no-slip solution.

Because this is an explicit marching scheme, there are no convergence criteria. Instead, the calculation is repeated with varying grid sizes to ensure grid independence. This algorithm is used with a starting $K_{1}$ value ranging from 100 to 200 , and $\Delta \eta$ varying from 0.0001 to 0.005 , to produce the results given.

\section{Computational Results for Fluid Flow}

The value of the stream function $f$ for $K_{1}$ ranging from 0 to 10 , which includes the entire slip-flow region, is show in Fig. 2. This figure shows that, for any value of $\eta$ in the boundary layer, $f$ decreases as the flow becomes more rarefied. Values of $u^{*}$ and $v^{*}$ are shown in Figs. 3 and 4. These results show that, at any given vertical position $\eta$ in the boundary layer, $u^{*}$ will increase, and $v^{*}$ will decrease, as the flow becomes more rarefied.

The nondimensional friction, or $f^{\prime \prime}(0)$, is shown in Fig. 5. Figure 5 shows the surprising result that the friction in the flow peaks not at the no-slip condition, but at a value of $K_{1}$ of approximately 0.50 . This is a result of the loss of self-similarity in the flow.

Closer inspection of the velocity contours shows that the $y$ velocity $v^{*}$ also peaks locally for values of $K_{1}$ less than 1.0. This gives some insight into the reason for this local increase in friction. As the flow proceeds along the plate, the value of $K_{1}$ decreases, and the slip 


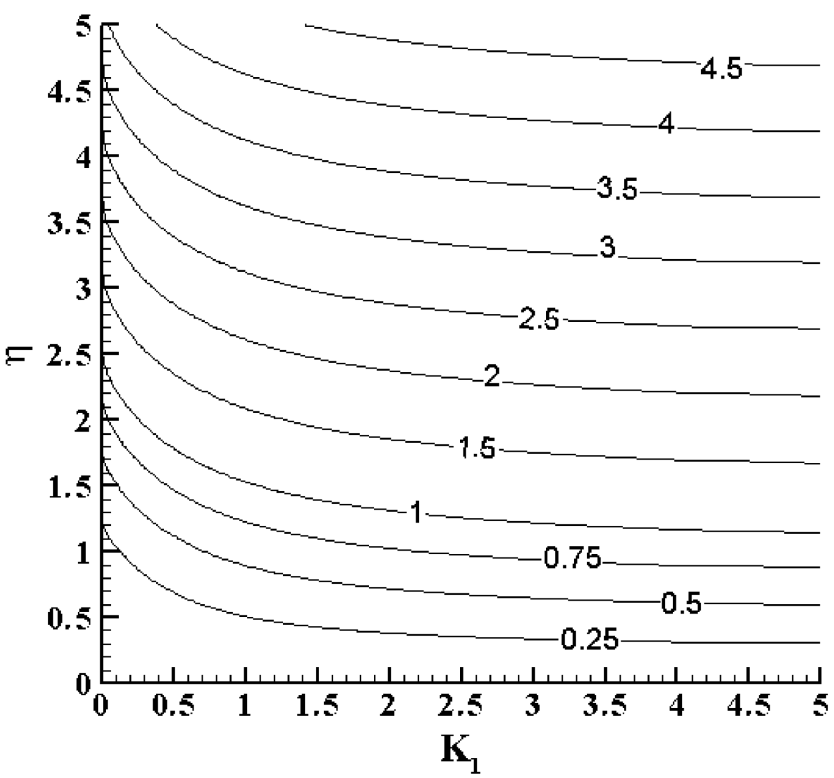

Fig. 2 Stream function as a function of position.

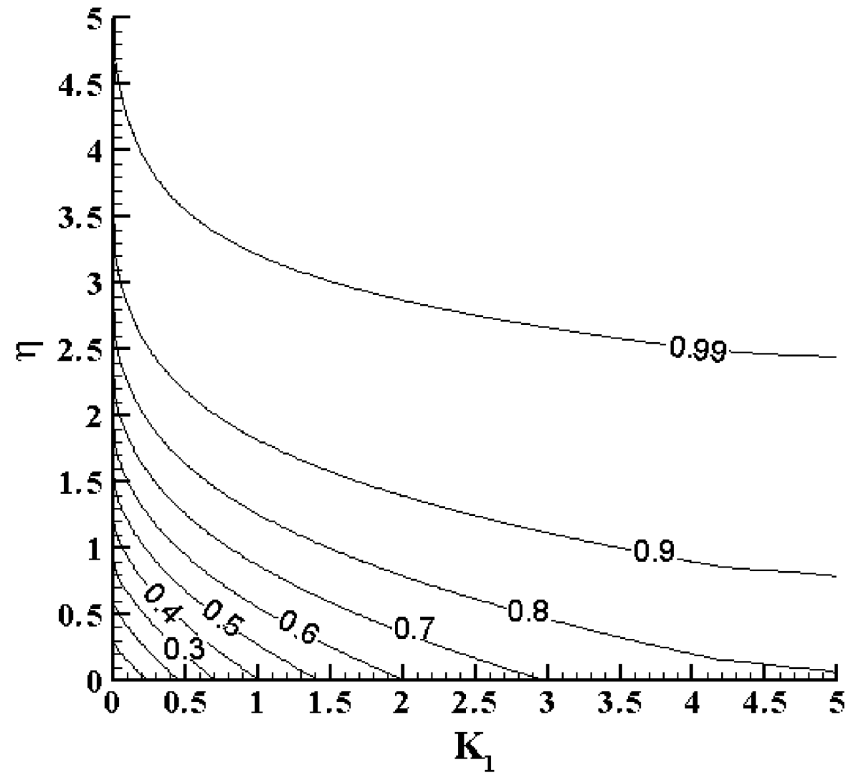

Fig. 3 Nondimensional $x$ velocity as a function of position.

velocity along the wall also decreases. Continuity requires that fluid moves away from the wall, leading to an increase in $v^{*}$.

The nondimensional wall shear stress $f^{\prime \prime}(0)$ is shown in Fig. 6. $f^{\prime \prime}(0)$ has a peak value of 0.4358 when $K_{1}$ is equal to 0.0467 . The two-dimensional effects cause a peak local friction of approximately $25 \%$ greater than the no-slip value. $f^{\prime \prime}(0)$ returns to the no-slip value as $K_{1}$ approaches 0 and asymptotically approaches zero as $K_{1}$ approaches infinity.

Figure 7 shows $f^{\prime}(0)$, or the nondimensional slip velocity, as a function of $K_{1}$. These results show that the wall velocity asymptotically approaches the freestream velocity as rarefaction increases.

As the Knudsen number approaches zero, $K_{1}$ also approaches zero, where the no-slip condition and the classical boundary layer solution are recovered. As the Knudsen number becomes large, $K_{1}$ approaches infinity, and the nondimensional slip velocity approaches 1, indicating $100 \%$ slip at the wall.

Figure 8 shows the normalized $x$ velocity profiles in the boundary layer for various values of $K_{1}$. One result that can be seen in Fig. 8 is that even as the wall velocity changes drastically, the overall

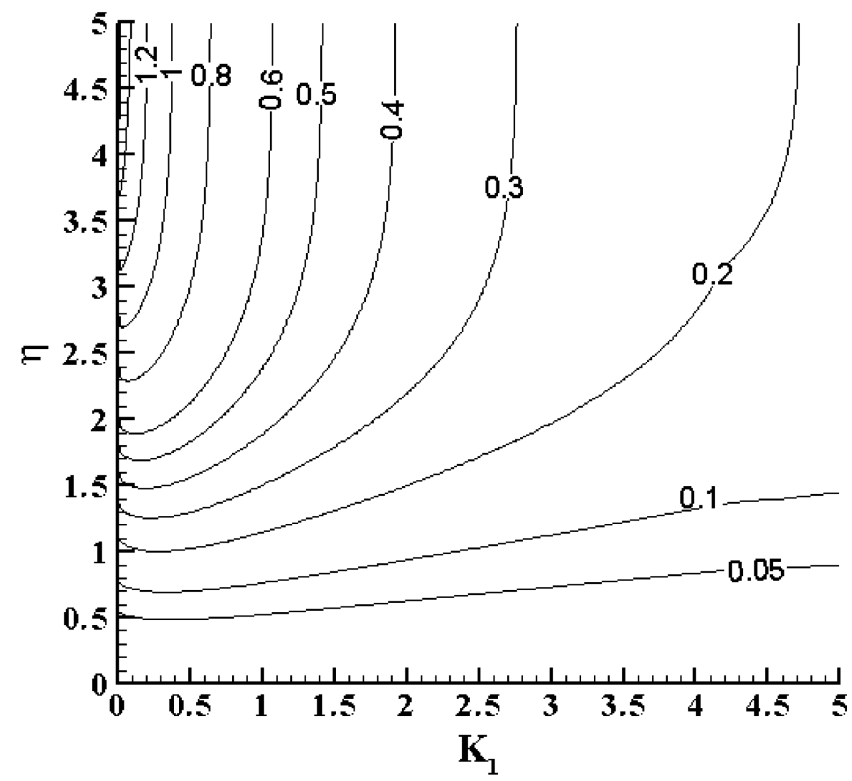

Fig. 4 Nondimensional $y$ velocity as a function of position.

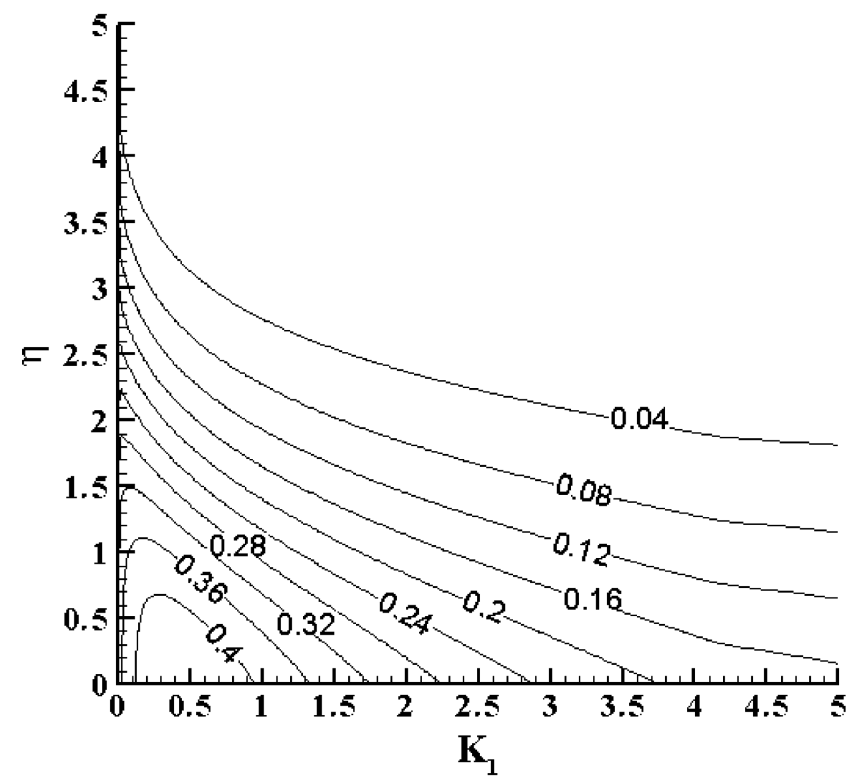

Fig. 5 Nondimensional friction as a function of position.

boundary layer thickness does not change as rapidly. The physical thickness of the boundary layer is given by Eq. (34):

$$
\delta_{99}=\eta_{99} \operatorname{Re}_{x}^{1 / 2} x
$$

For the no-slip boundary layer, $\eta_{99}$ is a constant with a value of 5.0. For a boundary layer with slip, $\eta_{99}$ varies along the plate. Figure 9 shows the value of $\eta_{99}$, where $u^{*}$ is equal to 0.99 , as a function of $K_{1}$. As the nondimensional wall slip velocity increases to greater than 0.99 , the boundary layer thickness becomes zero. This occurs at values of $K_{1}$ greater than 80

The definition of boundary layer thickness can be substituted into the definition of $K_{1}$ :

$$
K_{1}=\frac{(2-\sigma)}{\sigma} \frac{K n_{\delta}}{\eta_{99}}
$$

Because $\eta_{99}$ does not change by large amounts over the plate, this result suggests that slip-flow effects are a strong function of boundary layer thickness. 


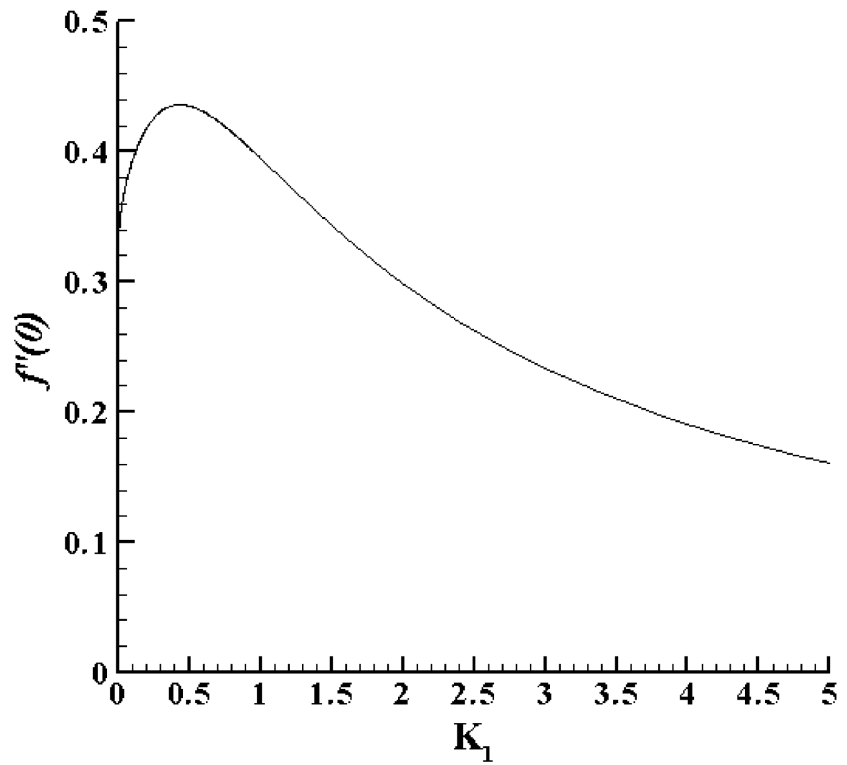

Fig. 6 Skin friction as a function of nonequilibrium.

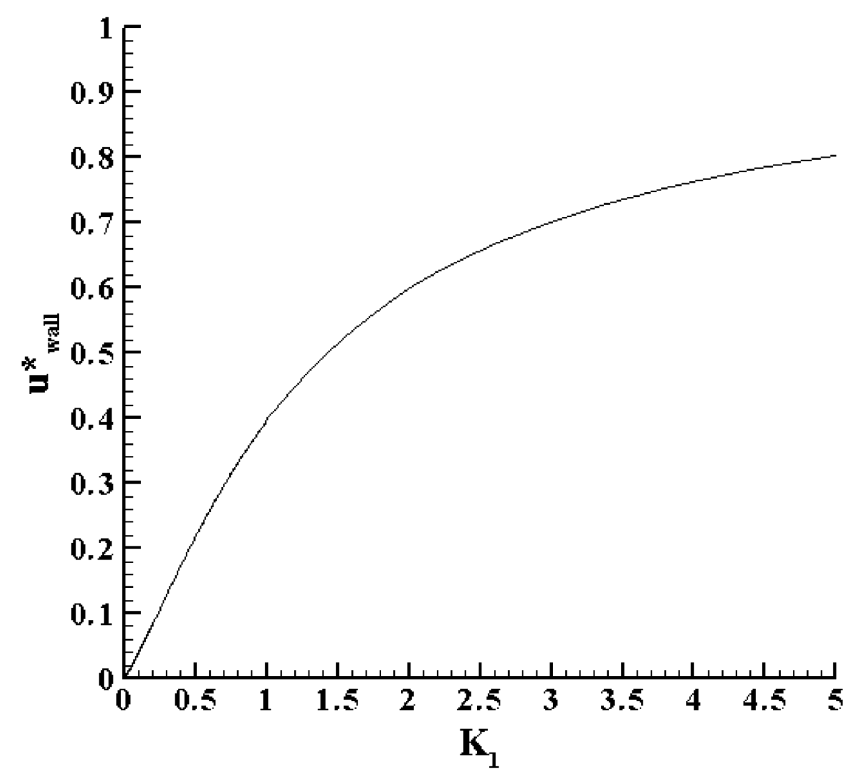

Fig. 7 Slip velocity as a function of nonequilibrium.

The displacement thickness and momentum thickness of the boundary layer will also vary with $K_{1}$. The displacement thickness, which measures the amount of the fluid displaced from the boundary layer, is defined as

$$
\delta^{*}=\int_{o}^{\infty}\left(1-\frac{u}{u_{o}}\right) \mathrm{d} y
$$

This can be converted into nondimensional coordinates:

$$
\frac{\delta^{*}}{\sqrt{u_{o} v / x}}=\int_{o}^{\infty}\left(1-f^{\prime}\right) \mathrm{d} \eta
$$

The momentum thickness, which measures the amount of momentum removed from the boundary layer, is defined as

$$
\theta=\int_{o}^{\infty} \frac{u}{u_{o}}\left(1-\frac{u}{u_{o}}\right) \mathrm{d} y
$$

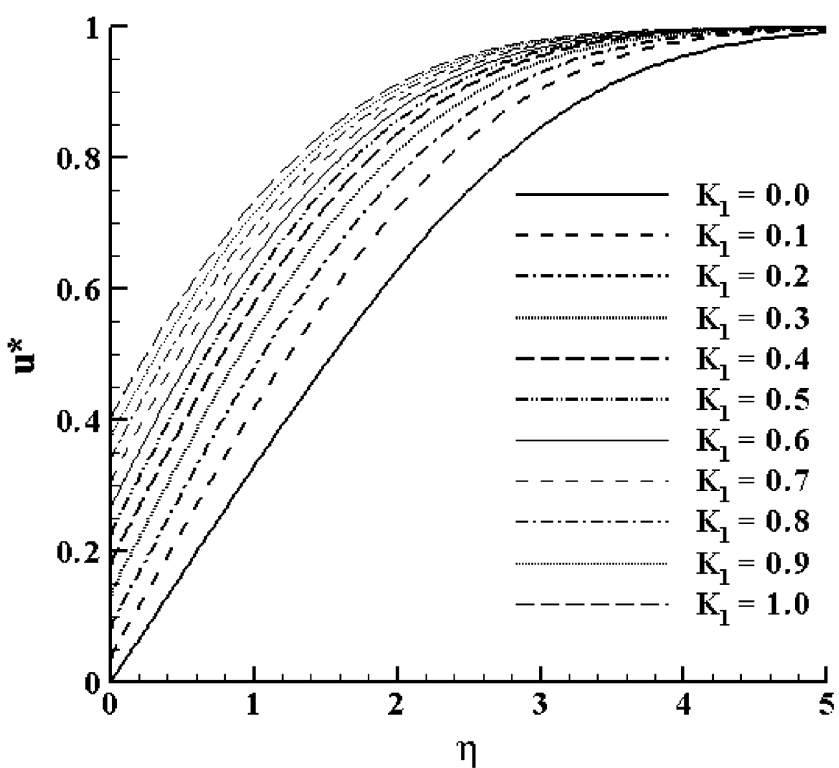

Fig. 8 Nondimensional $x$ velocity profiles.

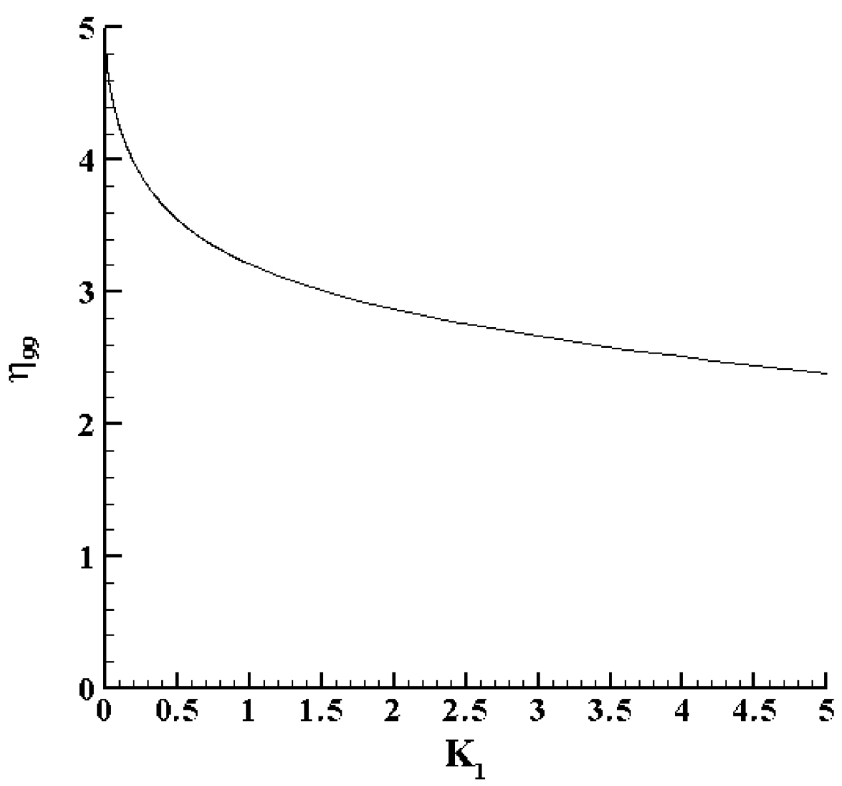

Fig. 9 Boundary layer thickness as a function of nonequilibrium.

This can be expressed in nondimensional form:

$$
\frac{\theta}{\sqrt{u_{o} v / x}}=\int_{o}^{\infty} f^{\prime}\left(1-f^{\prime}\right) \mathrm{d} \eta
$$

The nondimensional momentum thickness and displacement thickness as functions of $K_{1}$ are plotted in Fig. 10. These results show that both the velocity and momentum thickness decrease as $K_{1}$, and the amount of slip, increase. The results of this analysis can also be used to predict possible transition to turbulence within a rarefied boundary layer. A Reynolds number based on velocity thickness is calculated, as shown in Eq. (40):

$$
\operatorname{Re}_{\delta}=\frac{\rho \bar{u} \delta^{*}}{\mu}
$$

Previous researchers have found that laminar boundary layers become unstable when $R e_{\delta}$ approaches 520 [12]. of slip is to decrease $\delta^{*}$, this suggests that transition to turbulence may be delayed in a rarefied boundary layer. 


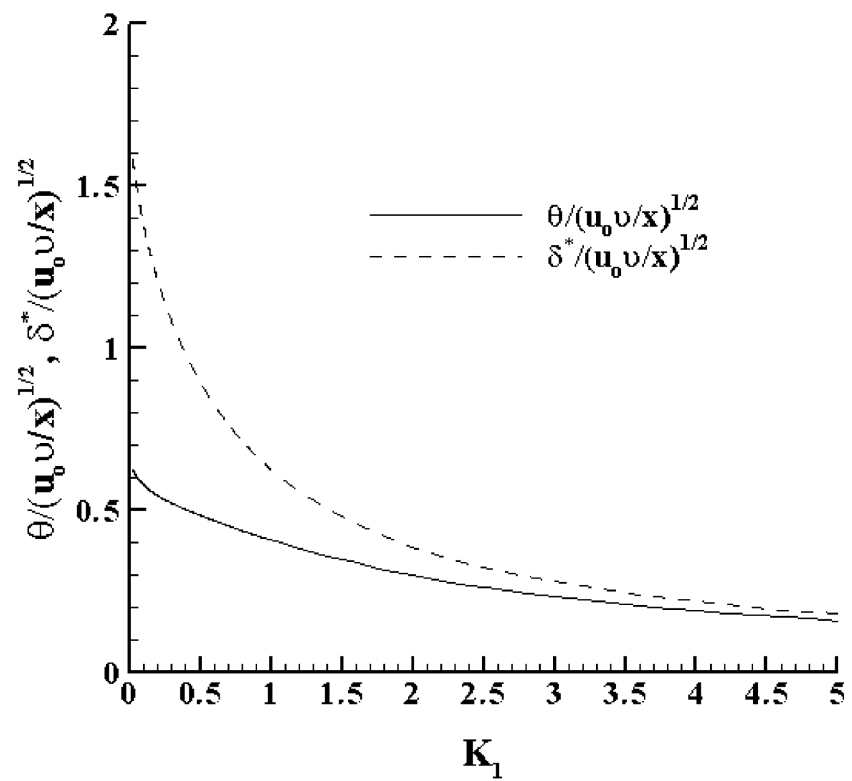

Fig. 10 Velocity and momentum thickness as functions of nonequilibrium.

\section{Calculation of Drag Force}

There are two engineering reasons to be interested in slip flow over a flat plate: decreases in skin friction and changes in heat transfer along the surface.

The wall friction $\tau_{w}$ for a laminar boundary layer is given by the expression below:

$$
\tau_{w}=\mu\left(\frac{\partial v}{\partial x}+\frac{\partial u}{\partial y}\right)=\mu \frac{\partial u}{\partial y}=\frac{\rho^{1 / 2} \mu^{1 / 2} u_{o}^{3 / 2}}{x^{1 / 2}} f^{\prime \prime}(0)
$$

The friction is proportional to the value of $f^{\prime \prime}(0)$ given in Fig. 6. Equation (41) can be integrated over the entire plate to obtain the net viscous drag force:

$$
F_{D}=\rho^{1 / 2} \mu^{1 / 2} u_{o}^{3 / 2} \int_{0}^{L} \frac{f^{\prime \prime}(0)}{x^{1 / 2}} \mathrm{~d} x
$$

The drag coefficient $C_{D}$ is defined as

$$
C_{D}=\frac{F_{D}}{L\left(\rho U^{2} / 2\right)}
$$

where $F_{D}$ is the drag per unit span of the airfoil.

For a flat plate with no slip, the drag coefficient can be obtained by integrating analytically, to obtain

$$
C_{D}=1.328 R e_{L}^{-1 / 2}
$$

For a flat plate with slip, the result must be obtained numerically. By substituting the definition of $K_{1}$ into (42), and performing the appropriate change of variables, we obtain

$$
C_{D}=4.0 R e_{L}^{-1 / 2} K_{1} \int_{K_{1}(L)}^{\infty} \frac{f^{\prime \prime}(0)}{K_{1}^{2}} \mathrm{~d} K_{1}
$$

The drag coefficient based on this integral is plotted in Fig. 11. The percent change in drag due to these effects compared to the no-slip solution is plotted in Fig. 12. These results show a slight increase in drag for slightly rarefied flows and then a large decrease in drag at higher Knudsen numbers.

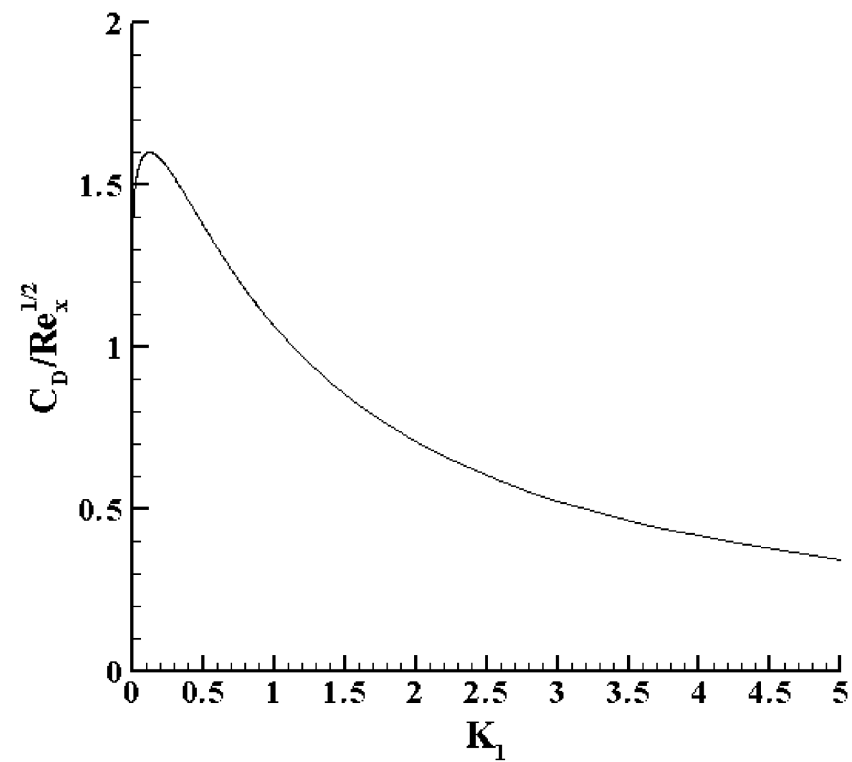

Fig. 11 Drag coefficient as a function of nonequilibrium.

\section{Heat Transfer in a Laminar Boundary Layer with Slip}

\section{A. Formulation of Boundary Layer Heat Transfer Equations with} Slip Flow

Once the velocity profile is calculated, the heat transfer in a slip flow can be calculated using the same approach that is used in the nonslip solution [13]. If viscous dissipation is neglected, then the equation for conservation of energy in a boundary layer with steady flow is given as

$$
u \frac{\partial T}{\partial x}+v \frac{\partial T}{\partial y}=\frac{\alpha}{\rho c_{p}} \frac{\partial^{2} T}{\partial y^{2}}
$$

A nondimensional temperature $T^{*}$ can be defined using the stream temperature $T_{o}$ and the surface temperature $T_{w}$ :

$$
T^{*}=\frac{T-T_{w}}{T_{o}-T_{w}}
$$

If we assume that $T^{*}$ is a function of $\eta$ and $K_{1}$, and apply the appropriate nondimensionalizations, Eq. (47) can be rewritten as

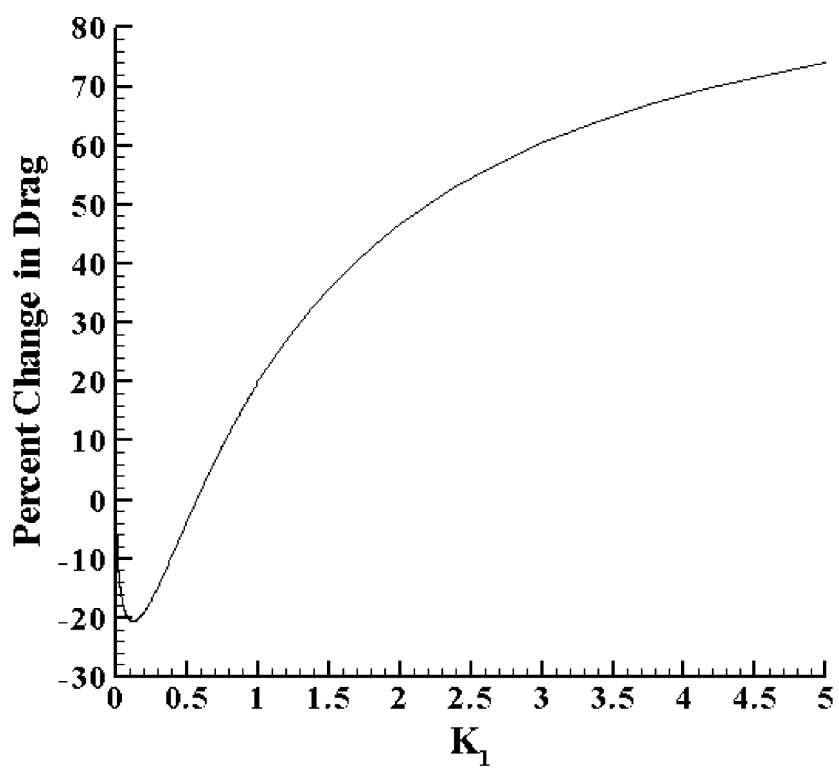

Fig. 12 Change in drag coefficient as a function of nonequilibrium. 


$$
0=\frac{\partial^{2} T^{*}}{\partial \eta^{2}}+\operatorname{Pr}\left[\left(\frac{\eta u^{*}}{2}-v^{*}\right) \frac{\partial T^{*}}{\partial \eta}+K_{1} u^{*} \frac{\partial T^{*}}{\partial K_{1}}\right]
$$

where $\operatorname{Pr}$ is the Prandtl number of the fluid.

The heat transfer equations are discretized using center-difference approximations in the $\eta$ direction, and a forward difference approximation in the $K_{1}$ direction, similar to the method used to solve the momentum equation. This results in the expression:

$$
T_{i-1, j}^{*}=[a+b] T_{i, j+1}^{*}+[1-2.0 a] T_{i, j}^{*}+[a+b] T_{i, j-1}^{*}
$$

where

$$
a=\frac{\Delta K_{1}}{\operatorname{Pr}^{*} K_{1}(\Delta \eta)^{2}}
$$

and

$$
b=\frac{\Delta K_{1}}{2 u^{*} K_{1} \Delta \eta}\left(\frac{\eta u^{*}}{2}-v^{*}\right)
$$

The stability criterion requires that all coefficients remain positive:

$$
\begin{gathered}
\Delta K_{1} \leq=\frac{\operatorname{Pr} K_{1} f_{i, j}^{\prime}(\Delta \eta)^{2}}{4.0} \\
\Delta \eta \leq \frac{4.0 P r}{f_{i, j}}
\end{gathered}
$$

The correct solution of the boundary layer heat transfer will require use of appropriate wall boundary conditions. The thermal boundary conditions for slip flow of a gas will differ from those of slip flow of a liquid, and the two cases will be considered separately.

\section{B. Heat Transfer with Slip Without a Temperature Jump}

Thermal boundary conditions for liquids at the microscale have not been extensively studied. Currently, calculations for heat transfer in liquids at the microscale assume that there is no thermal jump accompanying the velocity jump. If this is correct, then for liquids the temperature boundary condition at the wall will be the same as for nonslip flows:

$$
T(\eta=0)=T_{s}
$$

In nondimensional form, this becomes

$$
T^{*}(\eta=0)=0
$$

The boundary condition far from the wall will be

$$
T(\eta=\infty)=T_{\infty}
$$

In nondimensional form, this becomes

$$
T^{*}(\eta=\infty)=1
$$

At large values of $K_{1}$, the velocity approaches uniform flow, and the steady-state energy equation becomes

$$
\frac{\partial^{2} T^{*}}{\partial \eta^{2}}+\frac{P r}{2} \eta \frac{\partial T^{*}}{\partial \eta}=0
$$

This equation can be solved as an ordinary differential equation. The solution to this equation is used as the boundary condition at large values of $K_{1}$ :

$$
T^{*}\left(K_{1} \rightarrow \infty\right)=\operatorname{erf}[\sqrt{\operatorname{Pr}} \eta / 2]
$$

Using these boundary conditions, the heat transfer of a liquid boundary layer with wall slip is computed for Prandtl numbers of 1.0, 2.0, 3.0, 4.0, and 5.0. These values represent typical values for water and organic solvents near room temperature.

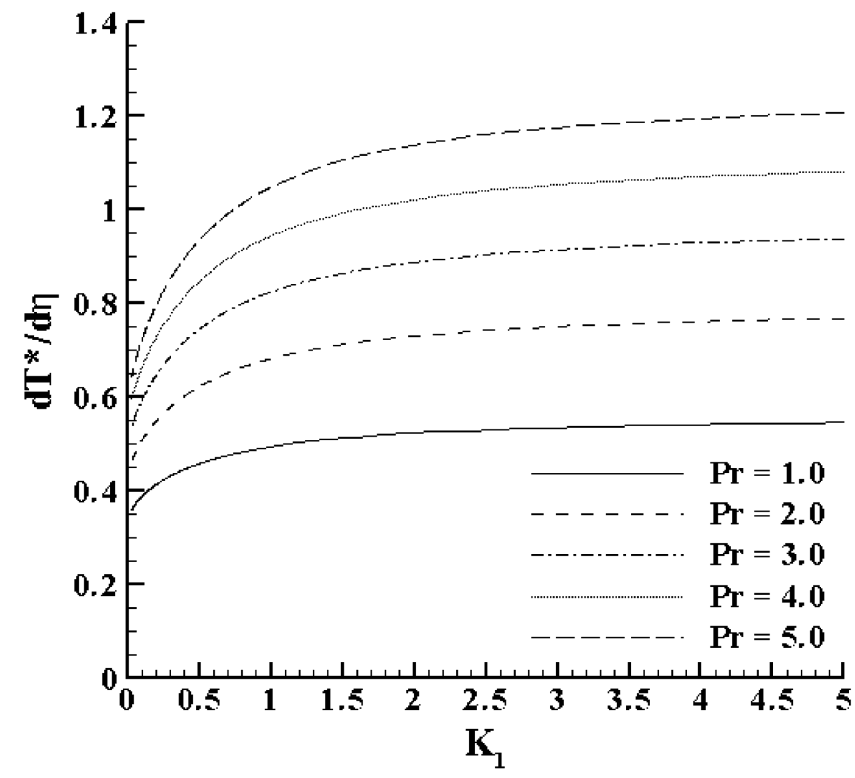

Fig. 13 Wall temperature gradient as a function of nonequilibrium for liquid flows.

The heat transfer coefficient $h$ is proportional to the local derivative at the wall:

$$
h=\frac{q_{s}^{\prime \prime}}{T_{w}-T_{o}}=-\left.\frac{T_{o}-T_{w}}{T_{w}-T_{o}} \alpha \frac{\partial T^{*}}{\partial y}\right|_{y=0}=\left.\alpha\left(\frac{\rho u_{o}}{\mu x}\right)^{1 / 2} \frac{\mathrm{d} T^{*}}{\mathrm{~d} \eta}\right|_{\eta=0}
$$

where $q_{s}^{\prime \prime}$ is the surface heat flux.

The derivative of the temperature at the wall as a function of $K_{1}$ is shown in Fig. 13. For the no-slip condition, the derivative at the wall is approximated by the following:

$$
\left.\frac{\mathrm{d} T^{*}}{\mathrm{~d} \eta}\right|_{\eta=0}=0.332 \operatorname{Pr}^{1 / 3}
$$

When compared with the no-slip results, Fig. 13 shows that for a liquid slip flow, the derivative at the wall, and the wall heat transfer, will increase as a result of slip.

To find the total heat transfer within the slip boundary layer, we integrate over the entire plate:

$$
\bar{h}=\frac{1}{L} \int_{o}^{L} k\left(\frac{u_{o}}{v x}\right)^{1 / 2} \frac{\mathrm{d} T^{*}(0)}{\mathrm{d} \eta} \mathrm{d} x
$$

Substituting the definition of $K_{1}$ into Eq. (62) transforms the equation into:

$$
\overline{N u}_{L}=2 R e_{L}^{1 / 2} K_{1} \int_{K_{1}(L)}^{\infty} \frac{\mathrm{d} T^{\prime}(0)}{K_{1}^{2}} \mathrm{~d} K_{1}
$$

This equation is used to calculate the average heat transfer over the entire surface, as shown in Fig. 14. These results can be compared with the no-slip value for the Nusselt number:

$$
\overline{N u}_{L}=0.664 \operatorname{Re}_{L}^{1 / 2} \operatorname{Pr}^{1 / 3}
$$

Figure 14 shows the average heat transfer over a flat plate increases when slip occurs.

\section{Heat Transfer in Gas Flows with Slip}

The same rarefied flow effects that produce velocity slip at the surface for gas flows will also produce a temperature jump [3]. The temperature jump boundary condition is given as 


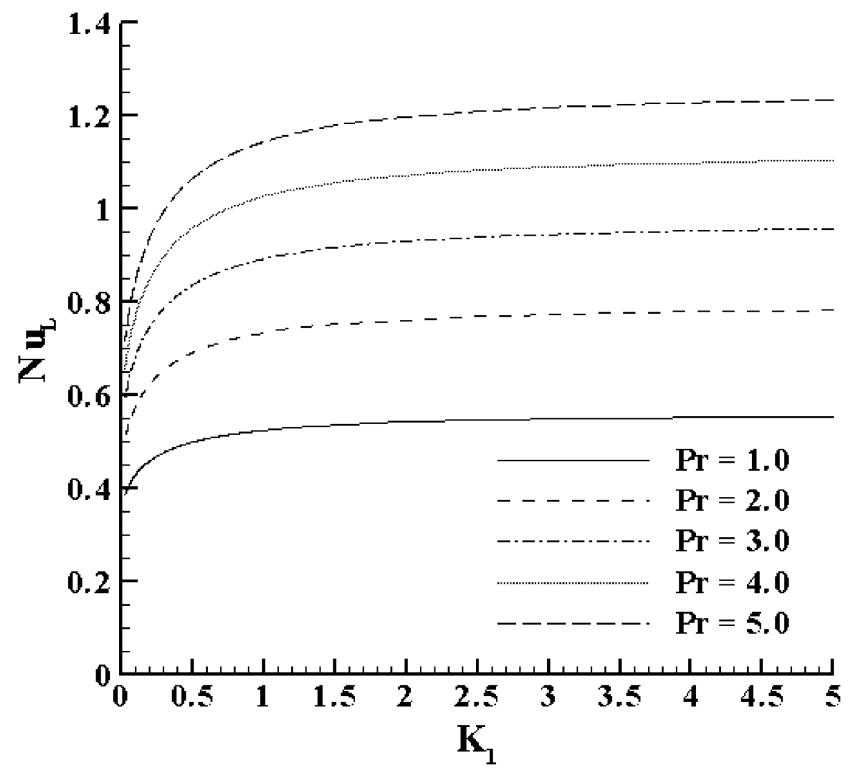

Fig. 14 Average Nusselt number as a function of nonequilibrium for liquid flows.

$$
T_{\mathrm{gas}}-T_{\mathrm{wall}}=\left.\frac{\lambda}{P r} \frac{2-\sigma_{T}}{\sigma_{T}} \frac{2 \gamma}{\gamma+1} \frac{\partial T}{\partial n}\right|_{\text {wall }}
$$

where $\sigma_{T}$ is the thermal accommodation coefficient.

Equation (65) can be nondimensionalized to obtain

$$
T^{*}(\eta=0)=\left.\frac{1}{P r} \frac{2-\sigma_{T}}{\sigma_{T}} \frac{2 \gamma}{\gamma+1} K n_{x} R e_{x}^{1 / 2} \frac{\partial T^{*}}{\partial \eta}\right|_{\text {wall }}
$$

Data on thermal accommodation coefficients are extremely limited [14], but the thermal accommodation coefficient and the momentum accommodation coefficients appear to be approximately equal. If this assumption is used, then Eq. (66) simplifies to

$$
T^{*}(\eta=0)=\left.\frac{1}{\operatorname{Pr}} \frac{2 \gamma}{\gamma+1} K_{1} \frac{\partial T^{*}}{\partial \eta}\right|_{\text {wall }}
$$

At large values of $K_{1}$, the temperature jump will become infinitely large, and the gradients at the wall will be negligible, giving a uniform temperature field:

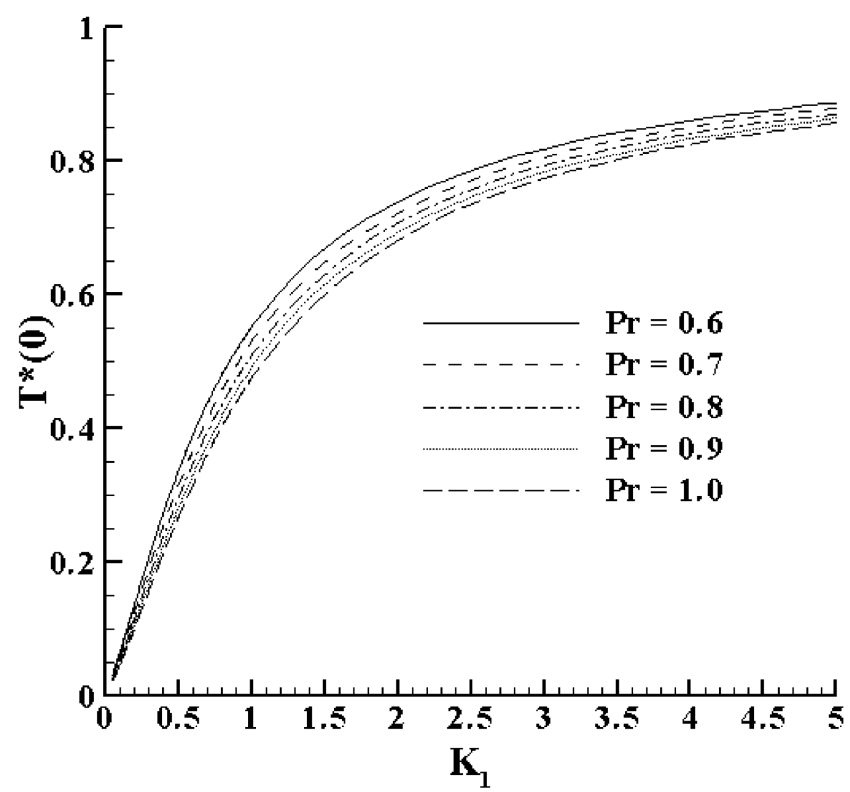

Fig. 15 Wall temperature as a function of nonequilibrium for $\gamma=7 / 5$.

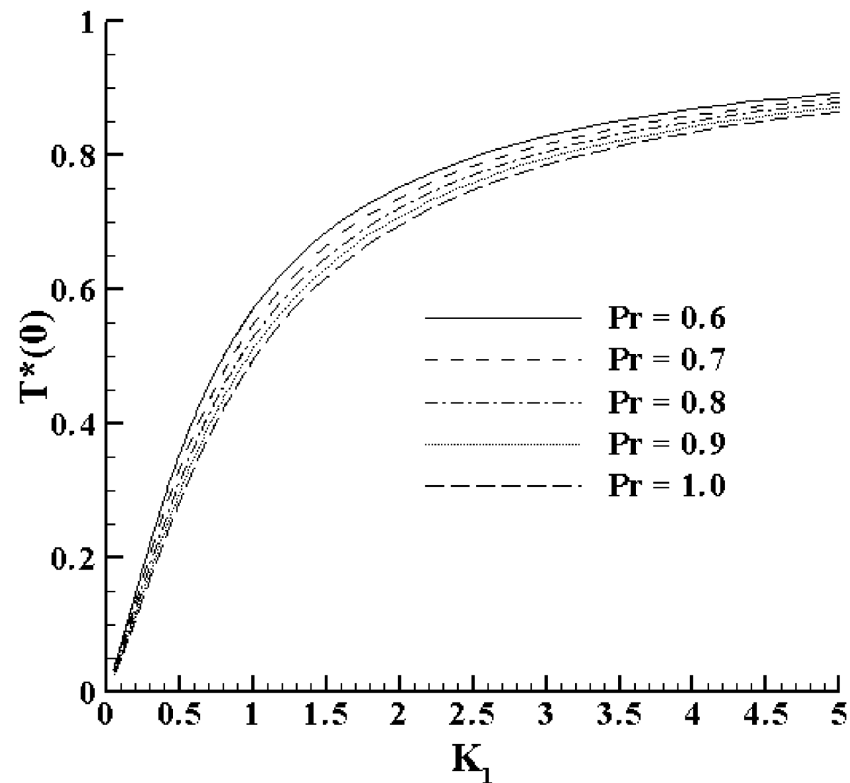

Fig. 16 Wall temperature as a function of nonequilibrium for $\gamma=5 / 3$.

$$
T^{*}\left(K_{1} \rightarrow \infty\right)=1
$$

Figures 15 and 16 show the wall temperature as a function of $K_{1}$ and $\operatorname{Pr}$ for values of $\gamma$ of $7 / 5$ for a diatomic gas and 5/3 for a monatomic gas. These results show the temperature jump is a substantial percentage of the temperature difference for the flow in the slip-flow regime.

Figures 17 and 18 show the wall temperature derivative as a function of $K_{1}$ and $\operatorname{Pr}$ for values of $\gamma$ of $7 / 5$ and 5/3. These results show that the heat transfer increases with increased Prandtl number. The heat transfer also increases as the specific heat ratio decreases. However, this change is generally less than $1 \%$. These results also show that the local heat transfer peaks at a value of $K_{1}$ of approximately 0.5 . The peak location increases with increased Prandtl number and decreases with increased specific heat ratio.

When these results are compared with the no-slip results, it is clear that heat transfer at the wall will decrease in highly rarefied flows and increase under moderately rarefied conditions. For slightly rarefied flows, the increased velocity near the surface more than offsets the reduced heat transfer due to the temperature jump. For flows with

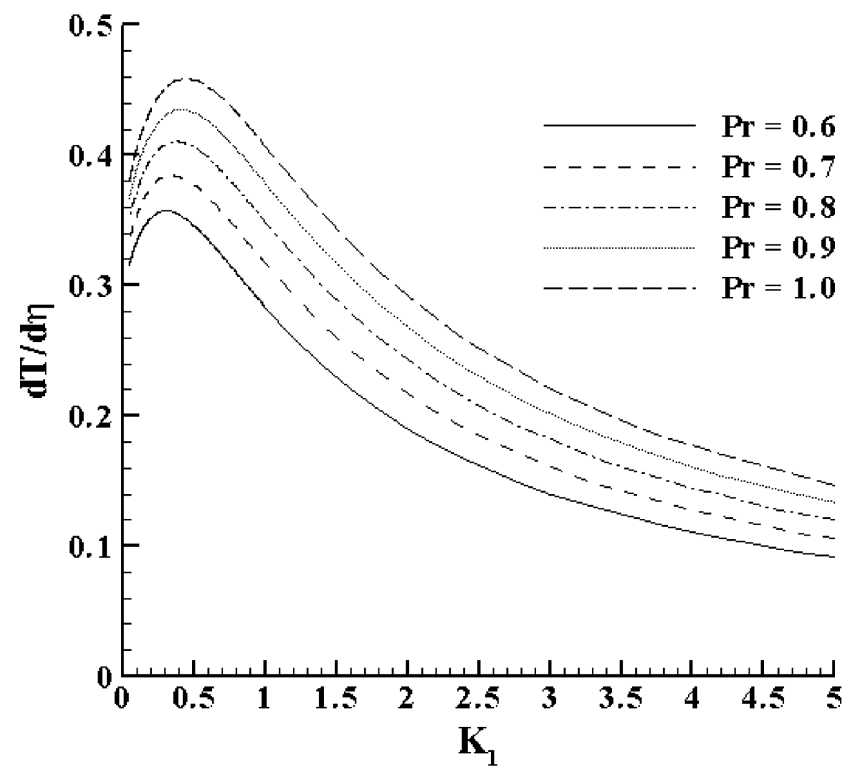

Fig. 17 Wall temperature gradient as a function of nonequilibrium for $\gamma=7 / 5$. 


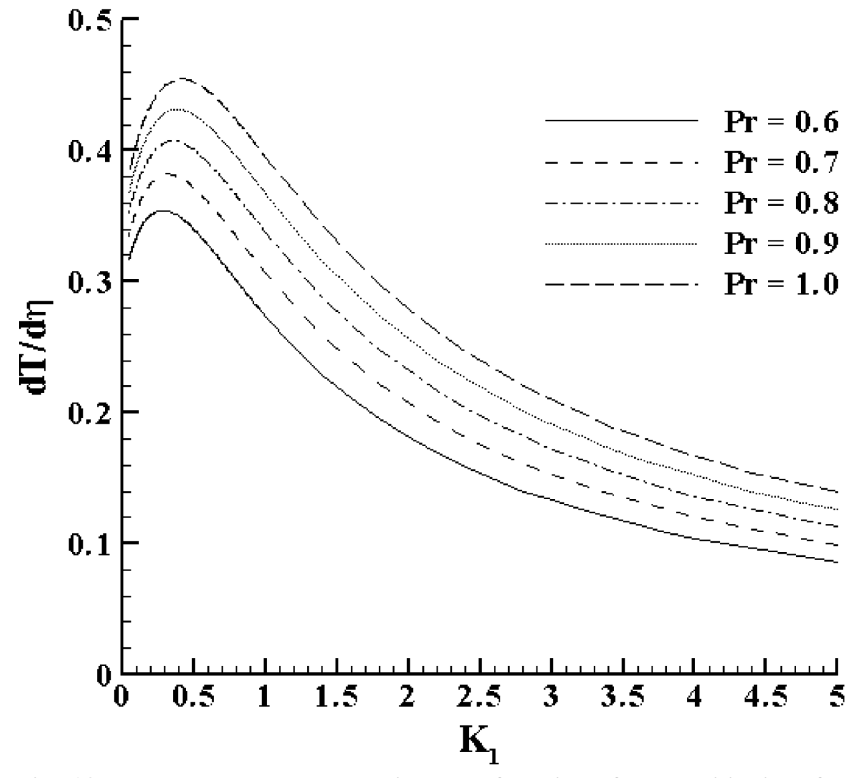

Fig. 18 Wall temperature gradient as a function of nonequilibrium for $\gamma=5 / 3$.

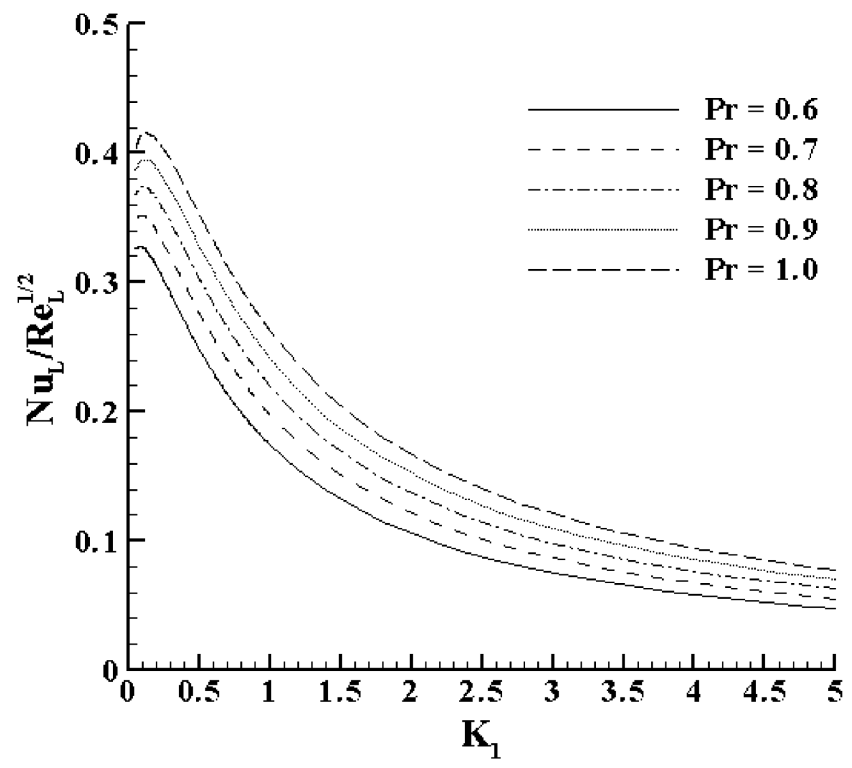

Fig. 19 Average Nusselt number as a function of nonequilibrium for $\gamma=7 / 5$.

larger Knudsen numbers, the heat transfer result is dominated by the temperature jump condition. The decreased heat transfer at higher Knudsen numbers agrees qualitatively with experimental results for heat transfer in heated cylinders at these Knudsen numbers [15].

Figures 19 and 20 show the average Nusselt number as a function of $K_{1}$ and $\operatorname{Pr}$ for values of $\gamma$ of $7 / 5$ and 5/3. These results again show an increase in heat transfer under slightly rarefied conditions and a decrease in heat transfer under highly rarefied conditions.

\section{Conclusion}

The equations for a laminar boundary layer can be solved nondimensionally in the presence of slip. The boundary layer loses the self-similarity of the no-slip Blasius solution. The loss of selfsimilarity leads to an increase in skin friction under some conditions. Other effects of slip included a thinner boundary layer and a more stable velocity profile.

The heat transfer in the boundary layer is also affected by the presence of slip. In liquid flows, where there is no temperature jump,

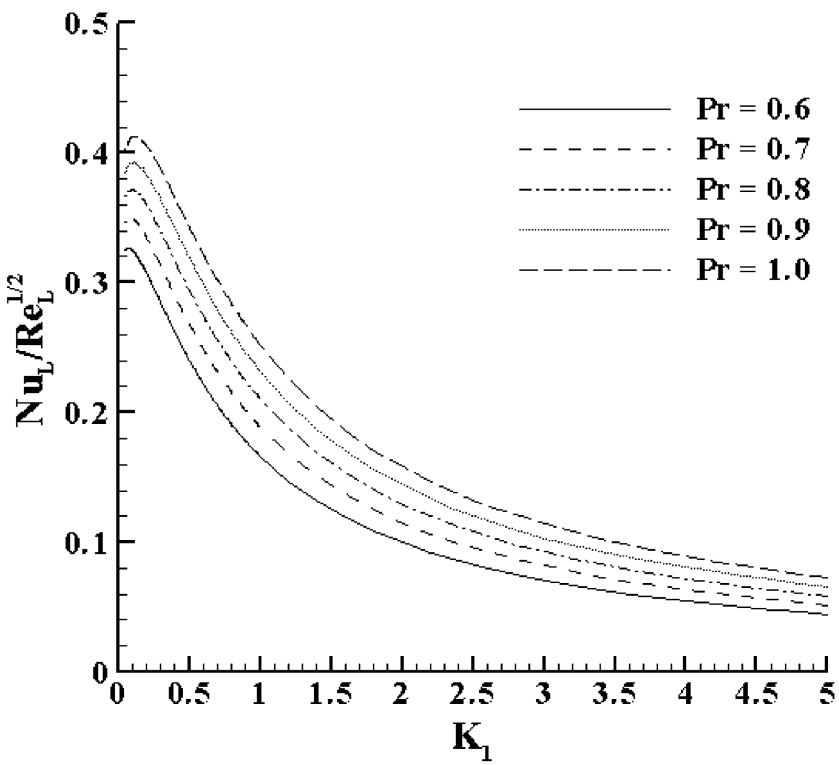

Fig. 20 Average Nusselt number as a function of nonequilibrium for $\gamma=5 / 3$.

the heat transfer increases as the slip velocity increases. In gas flows, a temperature jump condition is added and shown to scale with the velocity slip. The presence of the thermal jump condition decreases the heat transfer in the system. For slightly rarefied flows, increased fluid velocity near the wall more than offsets effects of the temperature jump, and the heat transfer will still be greater than for the no-slip case. For more rarefied flows, the heat transfer will decrease to values less than those of the no-slip case.

These results can be applied to analyze a variety of systems, including potential microdevice designs and flight in low-density atmospheres.

\section{Acknowledgment}

The authors gratefully acknowledge support for this work from the Air Force Office of Scientific Research through MURI Grant F49620-98-1-043.

\section{References}

[1] Ho, C. M., and Tai, Y. C., "Micro-Electro-Mechanical Systems (MEMS) and Fluid Flows," Annual Review of Fluid Mechanics, Vol. 30, 1998, pp. 579-612.

[2] Gad-el-Hak, M., "The Fluid Mechanics of Microdevices-The Freeman Scholar Lecture," Journal of Fluids Engineering, Vol. 121, No. 1, 1999, pp. 5-33.

[3] Maxwell, J. C., "On Stresses in Rarefied Gases Arising from Inequalities of Temperature," Philosophical Transactions of the Royal Society of London, Vol. 170, 1879, pp. 231-256.

[4] Wantanabe, K., Yanuar, and Mizunama, H., "Slip of Newtonian Fluids at Slid Boundary," JSME International Journal (B), Vol. 41, No. 3, 1998, pp. 525-529.

[5] Tretheway, D. C., and Meinhart, C. D., "Apparent Fluid Slip at Hydrophobic Microchannel Walls," Physics of Fluids, Vol. 14, No. 3, 2002, pp. L9-L12.

[6] Stone, H. A., Stroock, A. D., and Ajdari, A., "Engineering Flows in Small Devices," Annual Review of Fluid Mechanics, Vol. 36, 2004, pp. 381-411.

[7] Lin, T. C., and Schaaf, S. A., "Effect of Slip on Flow Near a Stagnation Point and in a Boundary Layer," NACA TN 2568, 1951.

[8] Kogan, M. N., Rarefied Gas Dynamics, Plenum Press, New York, 1969, pp. 386-400.

[9] Sun, Q., and Boyd, I. D., "Flat-Plate Aerodynamics at Very Low Reynolds Number," Journal of Fluid Mechanics, Vol. 502, March 2004, pp. 199-206.

[10] Blasius, H., "Grenzschichten in Flüssigkeiten mit kleiner Reibung," Zeitschrift für Mathematik und Physik, Vol. 56, No. 1, 1908, pp. 1-37. 
[11] White, F., Viscous Fluid Flow, 2nd ed., McGraw-Hill, New York, 1991, pp. 276-282.

[12] Schlichting, H., Boundary Layer Theory, 2nd ed., McGraw-Hill, New York, 1960, pp. 391-408.

[13] Incropera, F. P., Introduction to Heat Transfer, 3rd ed., Wiley, New York, 1990, pp. 386-399.
[14] Karniadakes, G. E., and Beskok, A., Microflows, Springer-Verlag, New York, 2002, pp. 51.

[15] Baldwin, L., Sandborn, V., and Laurence, J., "Heat Transfer from Transverse and Yawed Cylinders in Continuum, Slip, and Free Molecule Air Flows," Journal of Heat Transfer, Vol. 82, No. 2, 1960, pp. $77-88$. 